\title{
Injection Moulding of Oxide Ceramic Matrix Composites: Comparing Two Feedstocks
}

\author{
Maike Böttcher ${ }^{1, a^{*}}$, Daisy Nestler ${ }^{1, b}$, Jonas Stiller ${ }^{1, c}$ and Lothar Kroll ${ }^{1, d}$ \\ ${ }^{1}$ Department of Lightweight Structures / Polymer Technology, Chemnitz University of Technology, \\ 09107 Chemnitz, Germany \\ amaike.boettcher@mb.tu-chemnitz.de, bdaisy.nestler@mb.tu-chemnitz.de, \\ cjonas.stiller@mb.tu-chemnitz.de, dothar.kroll@mb.tu-chemnitz.de
}

\begin{abstract}
Keywords: ceramic-matrix composite, CMC, alumina, oxide ceramics, OCMC, ceramic injection moulding, CIM.
\end{abstract}

\begin{abstract}
Ceramic materials are suitable for use in the high temperature range. Oxide ceramics, in particular, have a high potential for long-term applications under thermal cycling and oxidising atmosphere. However, monolithic oxide ceramics are unsuitable for use in high-temperature technical applications because of their brittleness. Thin-walled, oxidation resistant, and hightemperature resistant materials can be developed by reinforcing oxide ceramics with ceramic fibres such as alumina fibres. The increase of the mechanical stability of the composites in comparison to the non-fibre reinforced material is of outstanding importance. Possible stresses or cracks can be derived along the fibre under mechanical stress or deformation. Components made of fibrereinforced ceramic composites with oxide ceramic matrix (OCMC) are currently produced in manual and price-intensive processes for small series. Therefore, the manufacturing should be improved. The ceramic injection moulding (CIM) process is established in the production of monolithic oxide ceramics. This process is characterised by its excellent automation capability. In order to realise large scale production, the CIM-process should be transferred to the production of fibre-reinforced oxide ceramics. The CIM-process enables the production of complicated component shapes and contours without the need for complex mechanical post-treatment. This means that components with complex geometries can be manufactured in large quantities.

To investigate the suitability of the injection moulding process for the production of OCMCs, two different feedstocks and alumina fibres (Nextel 610) were compounded in a laboratory-scale compounder. The fibre volume fractions were varied. In a laboratory-scale injection moulding device, microbending specimens were produced from the compounds obtained in this way. To characterise the test specimens, microstructure examinations and mechanical-static tests were done. It is shown that the injection moulding process is suitable for the production of fibre-reinforced oxide ceramics. The investigations show that the feedstocks used have potential for further research work and for future applications as material components for high-temperature applications in oxidising atmospheres.
\end{abstract}

\section{Introduction}

Fibre-reinforced oxide ceramics (OCMC) have a very high potential as a lightweight construction material for future applications, especially in complex load collectives with high thermal, mechanical and corrosive loads, e.g. as turbine components for power generation, for hot gas ducts and long-life burner nozzles of furnace plants, in chemical plant construction as well as in the aerospace industry. The increase of the fracture toughness as well as the damage and load tolerance in comparison to the non-fibre-reinforced material is of outstanding importance. At present this material has been accessible only to products in the high-price segment (aerospace, etc.) through manual manufacture. The requirement for the expansion of the application areas is the development of a suitable reproducible mass production process. In order to be able to realise mass production, the injection moulding process (CIM-Ceramic Injection Moulding) established in the production of monolithic 
oxide ceramics should be transferred to the production of fibre-reinforced oxide ceramics. This process is characterised by its excellent automation. The CIM process enables the production of complicated component shapes and contours without the need for complex mechanical posttreatment. This means that components with complex geometries can be manufactured in large quantities. The raw material system required for the CIM process consists of an organic binder and ceramic powder and is known as feedstock. The addition of the organic binder gives the ceramic powder a quasi-plastic behaviour. Further requirements for the organic binder system include shape stability during debinding, good storability, high green body strength, good demouldability and high adhesion between binder and powder particles [1]. The usual high injection pressures $(\mathrm{p}>50 \mathrm{MPa})$ for the CIM process and the resulting high compression of the ceramic matrix represent a challenge for the production of OCMCs by injection moulding. However, the required increase of fracture toughness, damage and stress tolerance for OCMCs is achieved by adjusting the matrix porosity. Fibre-reinforced oxide ceramics with a porous matrix are also referred as weak matrix composites (WMC) [2, 3]. The matrix mainly performs the function of holding the fibres together. The mechanical behaviour is fibre dominated, since the force-transmitting function is primarily carried out by the fibre. In the case of a specimen with microcracks, whose ceramic matrix material has passed the load limit, the fibre can still hold the structure together. The forces are redirected or transferred from the matrix via the interface to the fibre. In this way a pseudoplastic behaviour is achieved.

In order to investigate the basic transferability of the CIM process for the production of OCMCs, alumina fibres are mixed with two different feedstocks as a reinforcing component in the compounding process and then injection moulded test specimens are produced. The microstructural and mechanical properties of these test specimens are investigated and compared with an OCMC benchmark material. The influence of the process conditions including sintering temperature and time as well as the influence of fibre shortening and matrix compression resulting from the manufacturing process will be discussed.

\section{Experimental}

Materials. Two different feedstocks were used to produce the OCMCs and their suitability for this process was investigated. First, a commercially available alumina-feedstock was used. This feedstock was developed for the production of dense monolithic ceramic components using the Ceramic Injection Moulding (CIM) process. In the following, this feedstock is called CIM-matrix. On the second hand, an oxide ceramic matrix developed for the formation of OCMC-prepregs was used as feedstock [4]. This material is particularly suitable for low sintering temperatures and is designed to produce porous structures required for weak matrix composites (WMC). This feedstock is now called the WMC-matrix. Thermogravimetric analyses have shown that both feedstocks consist of 85 wt.\% ceramic alumina powder and 15 wt.\% organic binder system. Nextel $610(3 \mathrm{M})$ alumina rovings with 400 filaments were used as reinforcing fibres. The fibre volume content was varied.

Compounding. The feedstock-material and the fibre components were mixed in a co-rotating twinscrew extruder HAAKE MiniLab II (Thermo Scientific). This microcompounder is suitable for compounding and extruding small sample quantities. The reinforcing fibre was added as chopped fibre. Table 1 shows the composition of the compounds and the compounding parameters.

Injection moulding. Samples were produced from the compounds obtained using the HAAKE MiniJet II (Thermo Scientific) injection moulding machine. The injection moulding parameters are listed in Table 1. 
Table 1: Compounding parameters and injection moulding parameters

\begin{tabular}{|c|c|c|c|}
\hline $\begin{array}{l}\text { Fibre volume } \\
\text { content } \varphi[\%]\end{array}$ & Matrix & $\begin{array}{l}\text { Compounding } \\
\text { parameters }\end{array}$ & Injection moulding parameters \\
\hline 0 & \multirow{4}{*}{ CIM-matrix } & \multirow{4}{*}{$\begin{array}{l}\mathrm{T}=165{ }^{\circ} \mathrm{C} \\
\mathrm{n}=100 \mathrm{~min}^{-1}\end{array}$} & $\mathrm{~T}_{\text {cylinder }}=160^{\circ} \mathrm{C}, \mathrm{T}_{\text {mould }}=60^{\circ} \mathrm{C}$, \\
\hline 10 & & & $\mathrm{p}=650 \mathrm{bar}, \mathrm{t}=2 \mathrm{~s}$ \\
\hline 20 & & & \\
\hline 30 & & & holding pressure: $\mathrm{p}=400 \mathrm{bar}, \mathrm{t}=7 \mathrm{~s}$ \\
\hline 20 & \multirow{2}{*}{ WMC-matrix } & $\mathrm{T}=90^{\circ} \mathrm{C}$ & $\mathrm{T}_{\text {cylinder }}=130^{\circ} \mathrm{C}, \mathrm{T}_{\text {mould }}=30^{\circ} \mathrm{C}$, \\
\hline 30 & & $\mathrm{n}=85 \mathrm{~min}^{-1}$ & $\mathrm{p}=120 \mathrm{bar}, \mathrm{t}=3 \mathrm{~s}$ \\
\hline
\end{tabular}

Debinding and sintering. The green bodies obtained by injection moulding are debinded in a first step. A large part of the organic binder is removed. A two-stage process is required for the test specimens based on the CIM-matrix. First, aqueous debinding is carried out at room temperature and then thermal debinding. For the specimens based on the WMC-matrix, aqueous debinding is not necessary. The subsequent sintering of the specimens is carried out at a temperature of $1200^{\circ} \mathrm{C}$ with a holding time of five or ten hours.

Characterisation of mechanical properties. The characterisation of the mechanical properties of the OCMCs was performed by a three-point bending test (Kammrath \& Weiss) according to DIN EN 658-3 [5]. The size of the specimens produced is $47 \mathrm{~mm} \times 8 \mathrm{~mm} \times 3 \mathrm{~mm}$. Five samples of each sample series were tested. The test speed was $5 \mu \mathrm{m} / \mathrm{s}$.

Analysis of the microstructure. For the characterisation of the microstructure of the specimens, microsections were prepared. Both, the longitudinal and cross sections were examined by light microscopy (digital microscope VHX-600D (Keyence)). In addition, both metallographic specimen and fracture surfaces were examined by scanning electron microscopy (SEM) with the Nova NanoSEM 200 (FEI). For sample preparation, the non-conductive surfaces were coated with a 15$20 \mathrm{~nm}$ thin platinum layer by sputtering. Further SEM images were taken with the LEO1455VP (ZEISS) scanning electron microscope. The non-conductive samples were coated with graphite.

\section{Results and Discussion}

Compounding and injection moulding. On the one hand, the composition of the feedstock material used is optimised for the production of monolithic ceramics in the CIM process (CIMmatrix). On the other hand, the WMC-matrix was developed for the formation of OCMC-prepregs. Therefore, the use of these materials in a compounding and injection moulding process with reinforcing fibres meets only the requirements profile of the feedstocks to a limited extent. The proportion of Nextel 610 fibres used was adapted to the viscosity of the matrix system used. The typical fibre volume content $\varphi$ of $40 \%$ for OCMCs could not be achieved. Due to the different composition of the organic binder and the associated rheological behaviour, only a fibre volume content $\varphi$ of $30 \%$ could be obtained for the CIM-matrix. The WMC-matrix is characterised by a lower viscosity compared to the CIM-matrix. Therefore, no WMC-matrix-specimens with a fibre volume content $\varphi$ lower than $20 \%$ could be produced. The compounding process in the co-rotating twin-screw extruder results in a significant shortening of the fibre. The results of the light microscope analysis showed fibre lengths of 45-422 $\mu \mathrm{m}$ (mean value: $140 \mu \mathrm{m}$ ) for the CIM-matrix. Fibre lengths of 22-368 $\mu \mathrm{m}$ were detected for the WMC-matrix (mean value: $64 \mu \mathrm{m}$ ). These fibre lengths are comparable with the fibre lengths given in the literature for injection moulded fibre reinforced thermoplastics [6]. In addition to the fibre length, the fibre orientation was studied using light microscopic images. In Fig. 1 (a) can be seen that the fibres are oriented parallel to each other in the direction of processing due to the injection moulding process. In the area of the sprue (Fig. 1 (b)), fibres are also visible which are aligned vertically to the flow direction. This is due to the turbulence 
caused by the process. The CIM-matrix appears very homogeneous. In contrast, the micrograph of the specimens with the WMC-matrix (Fig. 2) showed that the matrix appears more inhomogeneous due to light and dark areas. The appearance of large pores is particularly critical.
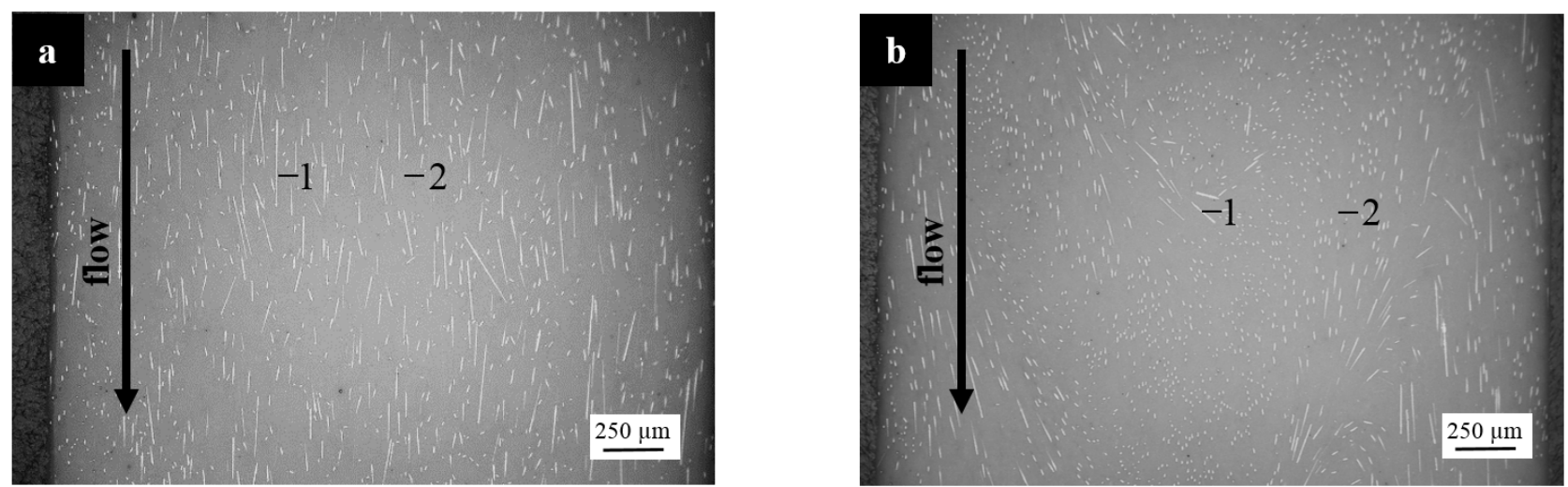

Figure 1: Light microscopic images of longitudinal sections of OCMC specimens

CIM-matrix, $\varphi=20 \%, 1$ - fibre, 2 - matrix

a) fibres oriented in flow direction; b) fibres oriented vertically to flow direction due to turbulences
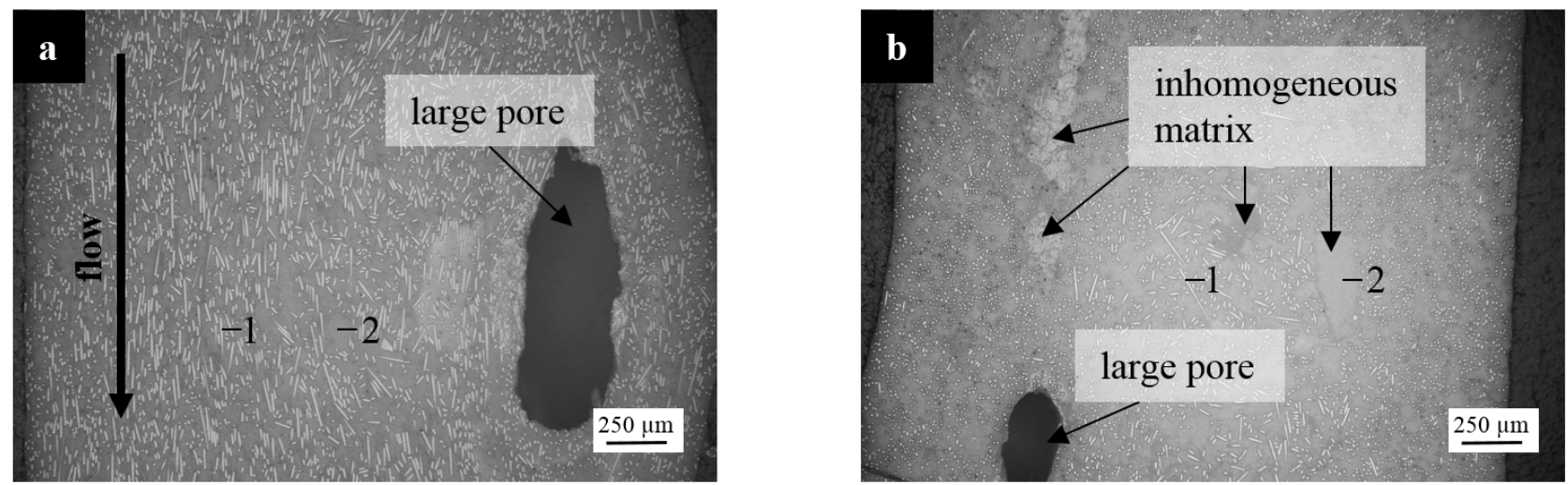

Figure 2: Light microscopic images of microsections of OCMC specimens

WMC-matrix, 1 - fibre, 2 - matrix; specimens show inhomogeneous matrix and large pores

a) $\varphi=30 \%$, longitudinal section; b) $\varphi=20 \%$, cross section

Sintering. For the sintering of the specimens, the temperature was limited to $1200{ }^{\circ} \mathrm{C}$. Higher temperatures should be avoided, as the mechanical properties of the Nextel 610 fibre will be degraded above a temperature of $1200{ }^{\circ} \mathrm{C}$. This is due to the coarsening of the ceramic grains of the fibres [7]. Some of the samples produced with the WMC-matrix could not keep their shape stability. Due to this fact and due to the inhomogeneity of the matrix and the presence of large pores in the specimens, only the CIM-matrix was examined for further experiments. The sintering temperature given by the manufacturer of the CIM-matrix is $1680{ }^{\circ} \mathrm{C}$ [8]. Due to the lower sintering temperature, the sintering time must be adapted. The first samples were sintered for five hours. The SEM images (see Fig. 3 (a) and (c)) show that these specimens have a sintered peripheral zone (a) and an unsintered core (c). In contrast to the peripheral zone unsintered alumina particles are still present in the sample core. The sintering time was therefore increased to ten hours. The SEM images (see Fig. 3 (b) and (d)) clearly show that the specimens were completely sintered due to the longer sintering time. The matrix appears densely sintered throughout. Pores in the size range of the alumina particles are recognisable. The number and size of the pores has decreased due to the longer sintering time. The uniform size distribution of the alumina particles results in a densely sintered matrix. 

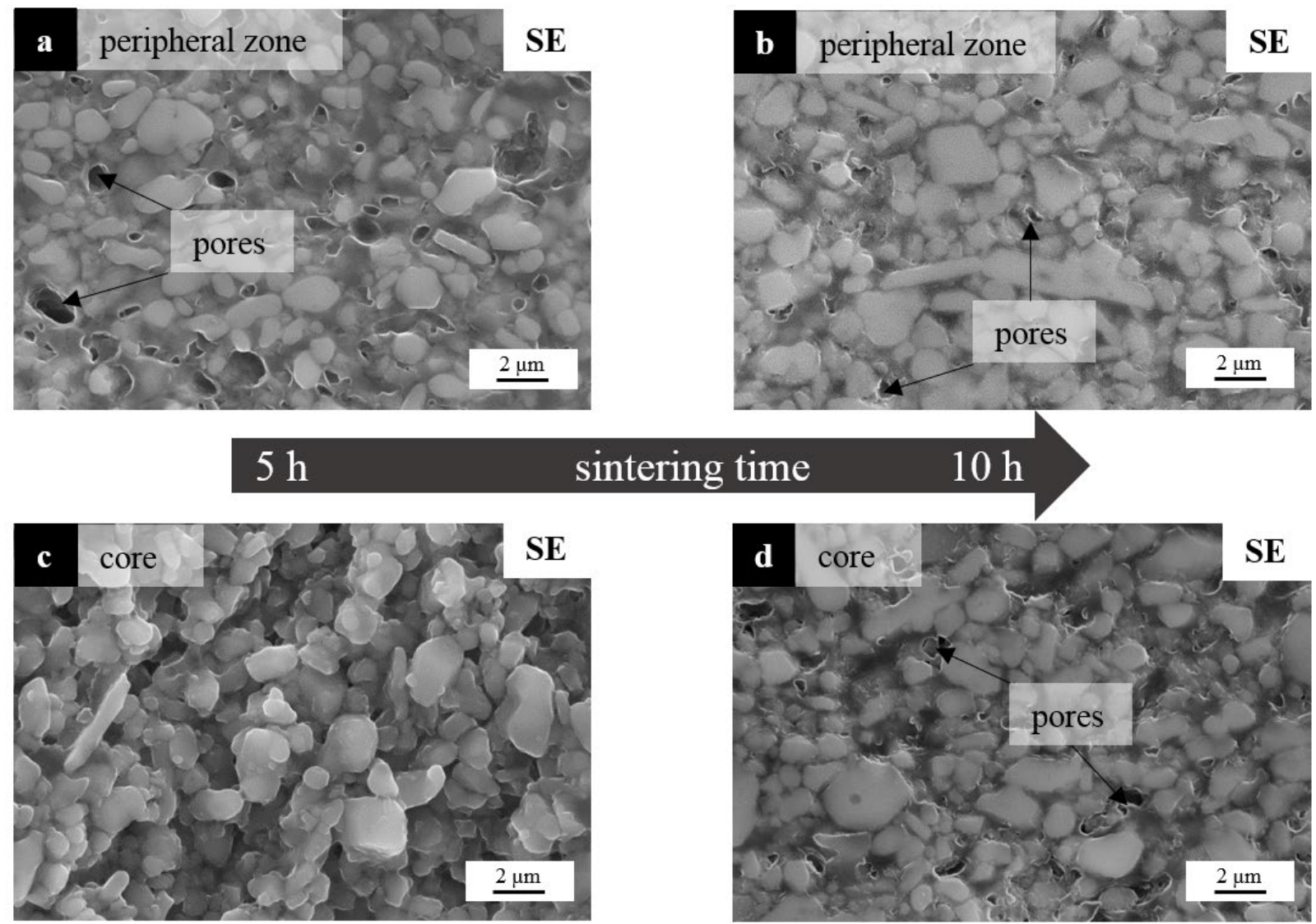

Figure 3: SEM-Images (SE) (LEO1455VP (ZEISS)) of cross sections of OCMC specimens, alumina CIM-matrix, influence of sintering time

a) peripheral zone of the specimen, sintered for $5 \mathrm{~h}$; b) peripheral zone of the specimen, sintered for $10 \mathrm{~h}$; c) core zone of the specimen, sintered for $5 \mathrm{~h}$; d) core zone of the specimen, sintered for $10 \mathrm{~h}$

Mechanical properties relating to the microstructure. In the three-point bending test, the Young's modulus and the flexural strength of the specimens produced were determined. Fig. 4 (a) shows that the Young's modulus was in the range of 38-52 GPa range at a sintering time of five hours. No significant influence of the matrix or the fibre volume content is detectable. In comparison, the Young's modulus of $153 \mathrm{GPa}$ was obtained for the samples with CIM-matrix which were sintered for ten hours. This significant increase is due to the already mentioned incomplete sintering of the specimens at a sintering time of five hours in comparison to completely sintered specimens after ten hours sintering time. Young's modulus of 50-92 GPa were given for commercially available continuous fibre-reinforced OCMCs (benchmark material) [9].

Fig. 4 (b) shows the measured flexural strengths. First, the specimens that have been sintered for five hours are analysed. The specimens with WMC-matrix have higher flexural strengths (93-112 MPa) than specimens with CIM-matrix (26-52 MPa). For the specimens with CIM-matrix, a trend can be seen that a higher fibre volume content leads to higher bending strengths. Increasing the sintering time to ten hours increases the flexural strength to $61 \mathrm{MPa}$. 


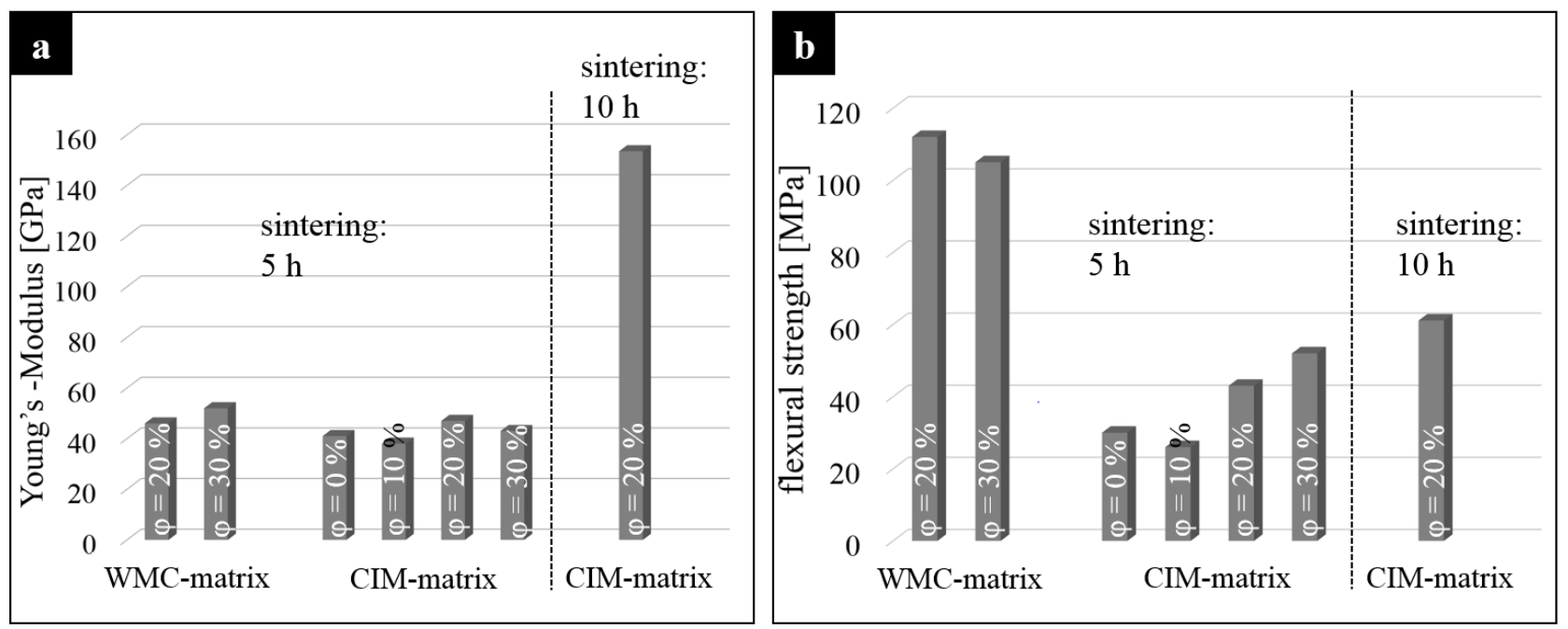

Figure 4: Three-point bending test of OCMC specimens

a) dependence of the Young's modulus on the matrix system, on the fibre volume content $\varphi$ and on the sintering time; $b$ ) dependence of the flexural strength on the matrix system, on the fibre volume content $\varphi$ and on the sintering time

The higher flexural strength of specimens with WMC-matrix compared to specimens with CIMmatrix may be due to the microstructure of the specimens analysed. In Fig. 5, SEM images of fracture surfaces of both specimens were compared. In the specimen analysed with the WMC-matrix (Fig. 5 (b)), a crack deflection is clearly visible on the fibre surface. In comparison to the specimen with CIM-matrix (Fig. 5 (a)), it can be seen that the particle size of the WMC-matrix is smaller than that of the CIM-matrix. How the particle size affects the mechanical behaviour has to be investigated in further experiments.
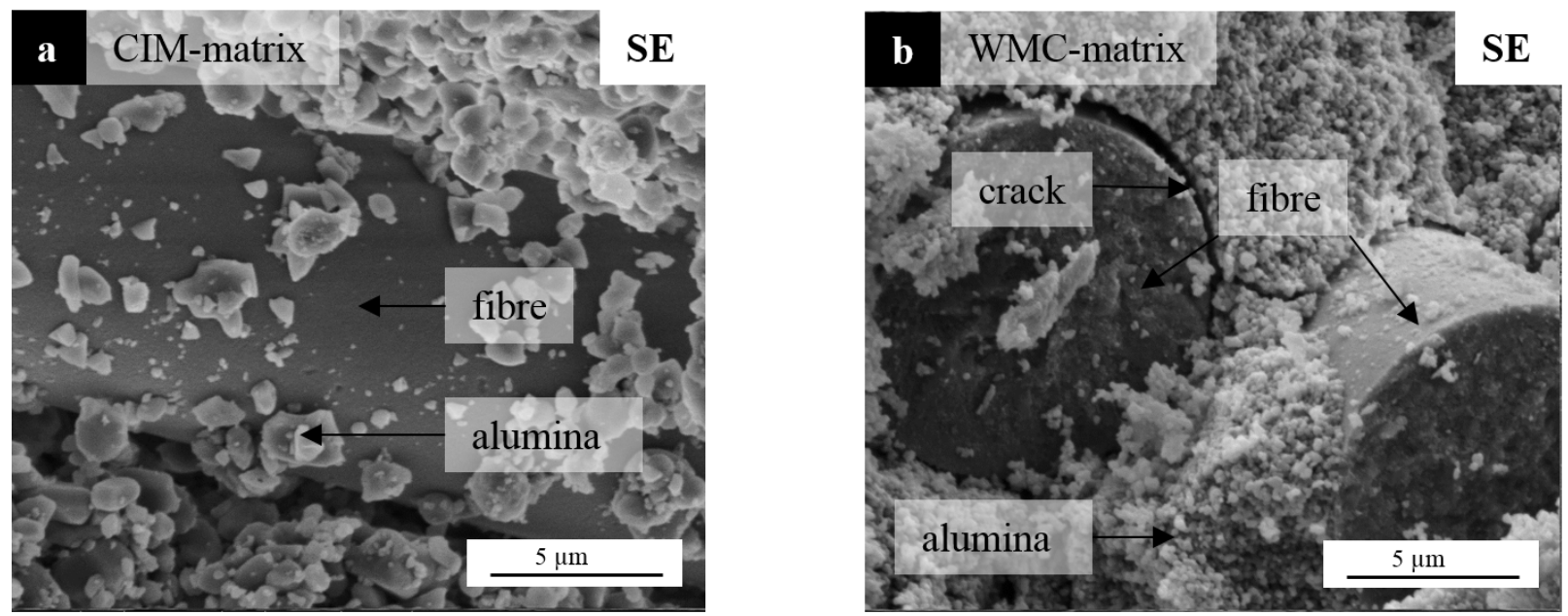

Figure 5: SEM-Images (SE) (Nova NanoSEM 200 (FEI)) of OCMC specimens, fracture surfaces, comparison of the matrix particle size

a) CIM-matrix, $\varphi=20 \%$; b) WMC-matrix, $\varphi=20 \%$, crack deflection at the fibre surface

Flexural strengths of 120-320 MPa are known for commercially available continuous fibrereinforced OCMCs (benchmark material) [9]. The test specimens produced by the CIM method thus do not achieve the mechanical properties of comparable benchmark-OCMCs. On the one hand, this is due to the lower fibre volume content. However, the strong shortening of the fibre by the compounding process has a more noticeable influence. In contrast, the reference materials have a continuous fibre reinforcement by fabric. Furthermore, the strong compression of the matrix due to the injection moulding process has a negative effect on the mechanical properties. In contrast to the benchmark-OCMCs with continuous fibre reinforcement by fabric, there is no porous matrix. As a 
result, the weak matrix concept cannot realised suffiently. Rather, the strong compression of the matrix results in a quasi-monolithic ceramic. This is also evident in the stress-strain diagram. The three-point bending tests performed on the specimens showed a typical stress-strain curve for monolithic materials, in which a brittle fracture occurs after an increase in stress with increasing bending.

\section{Conclusion}

The investigations described in this paper have shown that the CIM process is suitable for the production of OCMCs. Two different feedstocks were used. The investigations have shown that the CIM-matrix is more suitable for compounding and injection moulding than the WMC-matrix. When the WMC-matrix was used, inhomogeneous areas appeared in the test specimens, and it was also observed that the shape of the test specimens could not be kept during sintering. Since the sintering temperature was limited to $1200{ }^{\circ} \mathrm{C}$ to prevent degradation of the alumina fibres, the sintering time for the CIM-matrix was adapted. The analysis of microsection samples showed that the reinforcing fibres were directed in the direction of flow. This results in the fundamental possibility of aligning the fibres in the direction of the load. The mechanical characterisation shows that, on the one hand, the sintering time has a significant influence on the Young's modulus. The evaluation of the flexural strength showed that higher flexural strengths could be achieved with the WMC-matrix. This is due to the different microstructure of the matrices. The comparison with commercially available continuous fibre-reinforced OCMCs showed that the composites produced in the CIM process do not yet achieve the bending strength of this benchmark-OCMCs and the desired damage-tolerant behaviour. Further research and development work are necessary to achieve this goal. This includes adjusting the feedstock, increasing the fibre volume content, increasing the fibre length, adjusting a porous matrix and optimising the process parameters.

\section{Acknowledgement}

Thanks to INMATEC Technologies GmbH and Walter E.C. Pritzkow Spezialkeramik for providing the feedstock systems. Special thanks go to Mr. W. Pritzkow for realising the sintering tests and to Dr. H. Podlesak for his support in microstructure analysis (SEM).

\section{References}

[1] M. von Witzleben, Spritzgießen, in: W. Kollenberg (Ed.), Technische Keramik: Grundlagen, Werkstoffe, Verfahrenstechnik, second ed., Vulkan-Verlag GmbH, Essen, 2004, 451-458.

[2] M. Schmücker, P. Mechnich, All-oxide ceramic matrix composites with porous matrices, in: W. Krenkel (Ed.), Ceramic matrix composites: fiber reinforced ceramics and their applications. WileyVCH, Weinheim, 2008, 205-229.

[3] D. Koch, Microstructural Modeling and Thermomechanical Properties., in: W. Krenkel (Ed.), Ceramic matrix composites: fiber reinforced ceramics and their applications. Wiley-VCH, Weinheim, 2008, 231-259.

[4] P.O. Guglielmi, G.F. Nunes, M. Hablitzel, D. Hotza, R. Janssen, Production of Oxide Ceramic Matrix Composites by a Prepreg Technique, Materials Science Forum, 727 (2012), 556-561.

[5] DIN EN 658-3, Advanced technical ceramics - Mechanical properties of ceramic composites at room temperature - Part 3: Determination of flexural strength, (2002).

[6] H. Schürmann, Konstruieren mit Faser-Kunststoff-Verbunden, second ed., Springer, Berlin, 2005. 
[7] M. Schmücker, Faserverstärkte oxidkeramische Werkstoffe, Materialwissenschaft und Werkstofftechnik, 38 (2007), 698-704.

[8] Data sheets from Inmatec Technologies GmbH, URL: http://www.inmatecgmbh.com/download/2018/de/INMAFEED_K1010_Techn_Datenblatt.pdf; 24.01.2019

[9] Data sheets from Walter E.C. Pritzkow Spezialkeramik, URL: https://www.keramikblech.com/downloads/; 24.01.2019 